科 学通 报

\title{
哌啶氮氧自由基电子转移反应研究新进展 ${ }^{*}$
}

\author{
刘有成于灴郭失祥 \\ (兰州大学应用有机化学国家重点实验室、化学系,兰州 730000)
}

哌啶氮氧自由基是一类稳定的自由基, 其 $>\mathrm{N}-\mathrm{O}$ 官能团的邻位带有甲基叹代基的化合 物具有一定的化学惰性, 与许多试剂都不发生反应. 哌啶氮氧自由基的 4-位可连接多种取代 基,因而成为一种重要的自旋标记试剂,被广泛地应用于生物学、化学和物理学的研究中.

呱啶铖氧自由基的研究受到了许多化学家的重视,研究范围越来越广泛, 本文主要就哌啶 氮篻自由基的氧化还原性质及单电子转移反应的研究加以综述.

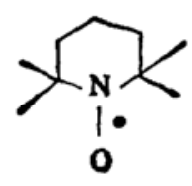

(I)

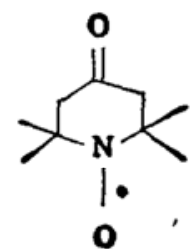

(n)<smiles></smiles>

(II)

哌啶氮氧自由基的未成对电子主要分布在 $\mathrm{N}$ 和 $\mathrm{O}$ 原子上, 氮原子构型为 s $p$ ' 杂化, $\mathrm{N}-\mathrm{O}$ 键和 C- N-C 键平面间有一定夹角(即离平面角)，4-位碳为 $s p^{3}$ 杂化的哌啶氮氧自由基的 构象为椅式, 4-位碳为 $s p^{2}$ 杂化的哌啶氮氧自由基的构象为扭曲椅式. 当氮原子相邻的碳上各 有两个甲基取代基时, 形成相当大的空间位阻, 所以 2, 2, 6, 6-四甲基哌啶氮篻自由基特别稳 定.

哌啶氮氧自由基可以作为电子给体或受体发生单电子氧化还原反应, 在乙腈溶液中哌啶 氮氧自由基可被电化学氧化为相应的锋锫盐, 在水溶液中的电化学氧化还原产物取决于介质 的 $\mathrm{pH}$ 值和电极材料. 氮氧自由基 $1 、 \mathrm{II}$ 和 $\mathrm{III}$ 的 $E_{\mathrm{P} / 2}$ 分别为 $0.29 \mathrm{~V}, 0.44 \mathrm{~V}$ 和 $0.34 \mathrm{~V}$. 哌啶氮氧自由基的电化学还原机理为 ${ }^{[1.23}$ :

$$
>\mathrm{N}-\mathrm{O} \underset{-\mathrm{e}^{-}}{\stackrel{+\mathrm{e}^{-}}{\rightleftharpoons}}>\mathrm{N}-\mathrm{O}^{-} \stackrel{\mathrm{H}^{+}}{\longrightarrow}>\mathrm{N}-\mathrm{OH}
$$

电化学氧化机理为 ${ }^{\mathrm{W}}$ :

$$
\begin{gathered}
>\mathrm{N}-\mathrm{O} \underset{+\mathrm{e}^{-}}{\stackrel{-\mathrm{e}^{-}}{\rightleftharpoons}}>\mathrm{N}^{+}=\mathrm{O} \\
>\mathrm{N}^{+}=\mathrm{O}+\mathrm{H}_{2} \mathrm{O} \rightleftharpoons \mathrm{N}-\mathrm{O}+\mathrm{H}^{+}+\mathrm{HO}^{\circ}
\end{gathered}
$$

1992-10-30 收稿, 1993-62-12收修改稙.

- 国家自然科学基金资助项目. 
在酸性条件下, 反应(3)受到抑制, 电极反应为单电子氧化可逆过程(2); 在强碱条件下, 可 能会发生下列反应 ${ }^{(3)}$ :

$$
\begin{gathered}
>\mathrm{N}^{+}=\mathrm{O}+\mathrm{OH}^{-} \rightarrow \mathrm{HO}-\underset{\mathrm{N}}{\mathrm{N}}=\mathrm{O} \\
\mathrm{HO}-\underset{\mathrm{N}}{\mathrm{N}}=\mathrm{O}+\mathrm{OH}^{-} \longrightarrow-\mathrm{O}-\underset{\mathrm{N}}{\mathrm{N}}=\mathrm{O}+\mathrm{H}_{2} \mathrm{O}
\end{gathered}
$$

Malinski ${ }^{[31}$ 在研究哌啶氮氧自由基的电化学反应时, 发现在 $\mathrm{pH}$ 为 12 时有新的自由基生 成, 并提出如下机理:
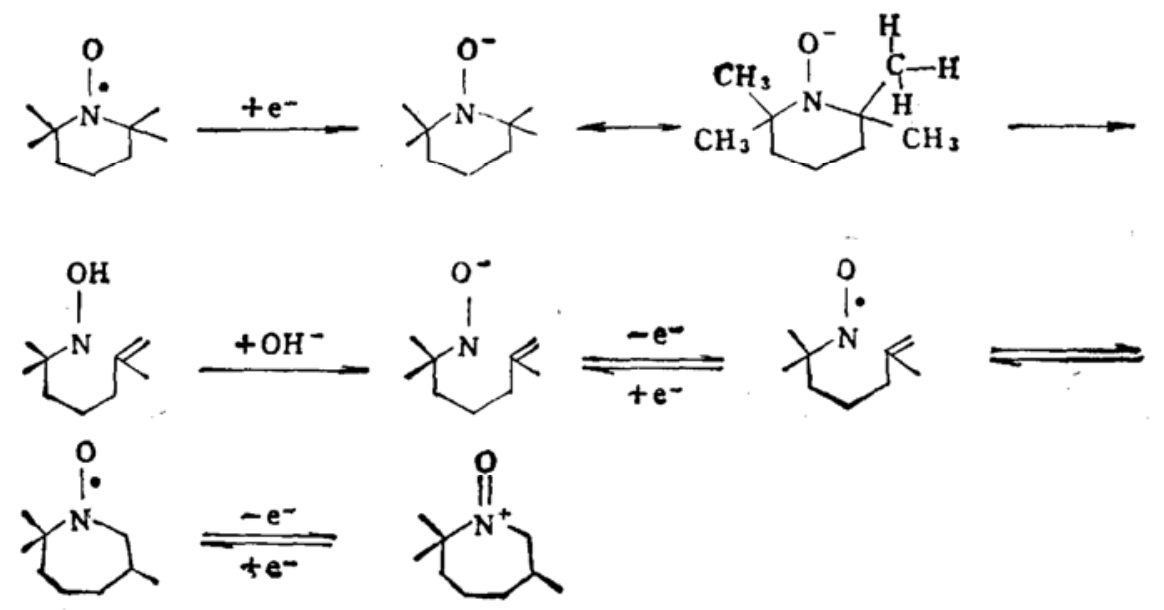

哌啶氮氧自由基在强酸性条件下发生歧化反应：

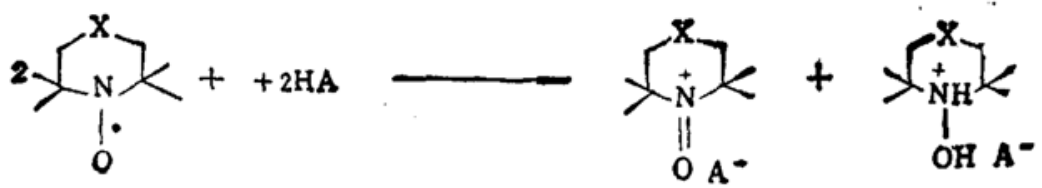

(式中 $\mathrm{HA}-\mathrm{H}_{2} \mathrm{SO}_{4}, \mathrm{HClO}_{4}, \mathrm{HBF}_{4} ; \mathrm{X}-\mathrm{CH}_{2}, \mathrm{C}-\mathrm{O}, \mathrm{CHOCOPh}$ ), 其机理为 ${ }^{\mathrm{\omega}}$ :

$$
\begin{gathered}
>\mathrm{N}-\mathrm{O}+\mathrm{H}^{+} \underset{k_{-1}}{\stackrel{k_{1}}{\rightleftharpoons}}>\mathrm{N}^{+\cdot}-\mathrm{OH} \\
2>\mathrm{N}^{+\cdot}-\mathrm{OH} \underset{k_{-2}}{\stackrel{k_{2}}{\rightleftharpoons}}>\mathrm{N}^{+}=\mathrm{O}+>_{\mathrm{NH}}^{\stackrel{+}{\mathrm{H}}-\mathrm{OH}}
\end{gathered}
$$

哌啶氮氧自由基和 1,5 -二氢黄素及 $\mathrm{N}^{3.5}$-二 二甲基-二氢黄素之间可发生电子转移反应, 反 应机理为<smiles>[R]N1c2cc(C)c(C)cc2Nc2c1n([R])c(=O)n([R4])c2=O</smiles>

FHH<smiles>[R]N1C(=O)C2C(Nc3cc(C)c(C)cc31)N([R])C(=O)N2[R]</smiles>

$\mathrm{ELH}_{2}$ 


$$
\begin{aligned}
& \mathrm{FlH}_{2}+>\mathrm{N}-\mathrm{O} \longrightarrow \mathrm{FlH} \cdot+>\mathrm{N}-\mathrm{OH} \\
& 2 \mathrm{FlH} \cdot \rightleftharpoons[\text { complex }] \rightleftharpoons \mathrm{Flox}+\mathrm{FlH}_{2} \\
& -\mathrm{FlH} \cdot+>\mathrm{N}-\mathrm{O} \longrightarrow \mathrm{Flox}+>\mathrm{N}-\mathrm{OH}
\end{aligned}
$$

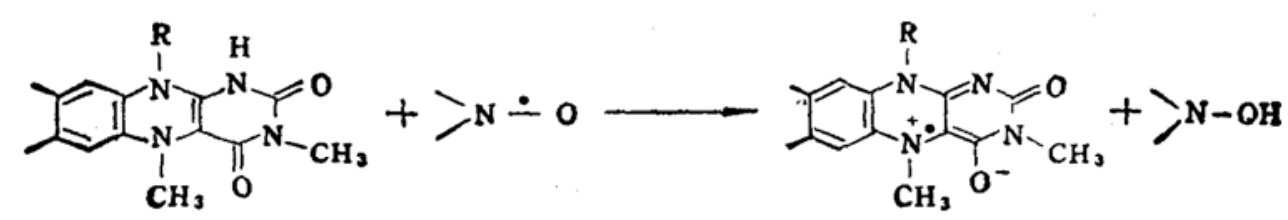

$\mathrm{F}_{3} \mathrm{HCH}_{3}$

$\mathrm{FiCH}_{3}$

当 $\mathrm{pH} \geqslant 9$ 时,生成的 $\mathrm{FlCH}_{\mathbf{3}}$ 进一步反应得到二氢黄素正离子.

氮氧自由基与脉冲辐射产生的自由基之间可发生电子转移反应.辐射产生的自由基, 如䍩 基自由基,与氮氧自由基按如下途径反应:

(1) 单电子转移反应

$$
>\mathrm{N}-\mathrm{O}+\mathrm{HO} \cdot \longrightarrow \mathrm{N}^{+}=\mathrm{O}+\mathrm{OH}^{-}
$$

当溶液的 $\mathrm{pH}$ 值很高时,

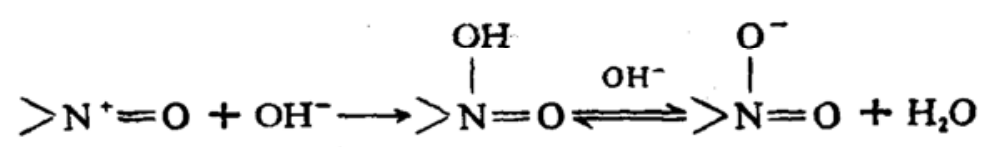

（2）自由基反应

$$
>\mathrm{N}-\mathrm{O}+\mathrm{HO} \longrightarrow \longrightarrow \mathrm{N}-\mathrm{O}-\mathrm{OH}
$$<smiles></smiles>

（3）氮氧自由基被溶剂化电子或氢原子还原,在中性或碱性介质中生成羟胺

$$
\begin{aligned}
& >\mathrm{N}-\mathrm{O}+\mathrm{e}_{\mathrm{a}_{\mathrm{q}}}^{-} \longrightarrow \mathrm{N}-\mathrm{O}^{-} \\
& >\mathrm{N}-\mathrm{O}^{-}+\mathrm{H}_{\mathrm{a}_{\mathrm{q}}^{+}} \longrightarrow>\mathrm{N}-\mathrm{OH} \\
& >\mathrm{N}-\mathrm{OH}+\mathrm{H}_{\mathrm{aq}_{\mathrm{q}}}^{+} \longrightarrow>\mathrm{NH}-\mathrm{OH} \\
& >\mathrm{N}-\mathrm{O}+\mathrm{H}^{\cdot} \longrightarrow>\mathrm{N}-\mathrm{OH}
\end{aligned}
$$

（4）氮氧自由基被 $\alpha$-楼基自由基还原

$$
>\mathrm{N}-\mathrm{O}+>\mathrm{C} \cdot-\mathrm{OH} \longrightarrow>\mathrm{N}-\mathrm{OH}+>\mathrm{C}=\mathrm{O}
$$

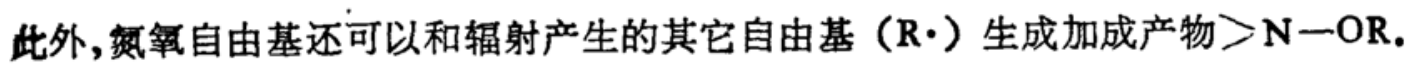

氧氧自由基和电子受体醌类化合物 $(Q)$ 和紫精 (Viologen) $V^{2+}$ 反应, 机理如下:

$$
\begin{aligned}
& \mathrm{H}_{2} \mathrm{O} \rightarrow \longrightarrow \mathrm{e}_{\mathrm{si}}^{-}, \mathrm{HO}^{-}, \mathrm{H}^{+},\left(\mathrm{H}_{2}, \mathrm{H}_{2} \mathrm{O}_{2}\right) \\
& \mathrm{e}_{\mathrm{aq}^{-}}^{-}+\mathrm{V}^{2+} \longrightarrow \mathrm{V}^{+} \\
& \mathrm{HO} \cdot(\mathrm{H} \cdot)+\mathrm{HCO}_{2}^{-} \longrightarrow \mathrm{H}_{2} \mathrm{O}\left(\mathrm{H}_{2}\right)+\mathrm{CO}_{i}^{-} \\
& \mathrm{CO}_{i}^{-}+\mathrm{V}^{2+} \longrightarrow \mathrm{CO}_{2}+\mathrm{V}^{+}
\end{aligned}
$$




$$
\begin{aligned}
& \mathrm{e}_{24}\left(\mathrm{CO}_{2}^{-}\right)+\mathrm{Q} \longrightarrow \mathrm{Q}^{-}\left(\mathrm{CO}_{2}\right) . \\
& \mathrm{V}^{+}\left(\mathrm{Q}^{-}\right)+>\mathrm{N}-\mathrm{O} \rightleftharpoons \mathrm{V}^{2+}(\mathrm{Q})+>\mathrm{N}-\mathrm{O}^{-} \stackrel{+\mathrm{H}^{+}}{\underset{-\mathrm{H}^{+}}{\longrightarrow}}>\mathrm{N}-\mathrm{OH}
\end{aligned}
$$

维生素 C 是生物体内的一种常见的还原剂, 近年来关于维生素 C 与氮篻自由基反应的报 道很多. 大量研究表明, 氮氧自由基的结构影响其与维生素 $\mathrm{C}$ 的反应速度. 六员环氮氧自由 基被还原的速度大于五员环氮氧自由基、六员环羟胺被重新氧化的速度也大于五员环。取代 基也影响反应速度,但不如环的影响大 ${ }^{[4]}$. 维生素 $\mathrm{C}$ 的还原形式是单去质子维生素 $\mathrm{C}$ 负离子。

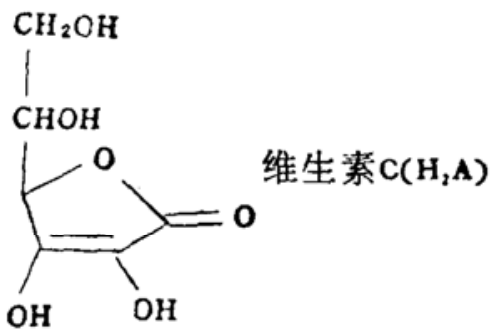

维生素 C 与哌啶氮氧自由基反应动力学的研究支持如下机理：

$$
\begin{aligned}
& \mathrm{H}_{2} \mathrm{~A} \mathrm{HA}^{-}+\mathrm{H}^{+} \\
& \mathrm{HA}+>\mathrm{N}-\mathrm{O} \longrightarrow \mathrm{HA} \cdot+\mathrm{N}-\mathrm{O}^{-} \\
& \mathrm{HA} \rightleftharpoons \mathrm{A}+\mathrm{H}^{+} \\
& \mathrm{A}^{-}+>\mathrm{N}-\mathrm{O} \longrightarrow \mathrm{N}-\mathrm{O}^{-}+\mathrm{A} \\
& >\mathrm{N}-\mathrm{O}^{-}+\mathrm{H}_{2} \mathrm{O} \longrightarrow \mathrm{N}-\mathrm{OH}+\mathrm{OH}^{-} \\
& \mathrm{A}+\mathrm{H}_{2} \mathrm{O} \longrightarrow \text { 水合物 } \\
& 2 \mathrm{~A}^{-} \longrightarrow \mathrm{A}+\mathrm{A}^{2-} \\
& \mathrm{HA}^{-} \longrightarrow \mathrm{A}^{2-}+\mathrm{H}^{+}
\end{aligned}
$$

哌啶氮氧自由基与 $d, 1-$ 半睄氨酸反应, 在酸性缓冲溶液中生成 $d, 1$-磺基丙氨酸, 在碱性 缓冲溶液中生成 $d, 1$-胱氨酸,氮氧自由基被还原成羟胺。在碱性条件下的反应按下列机理进 行:

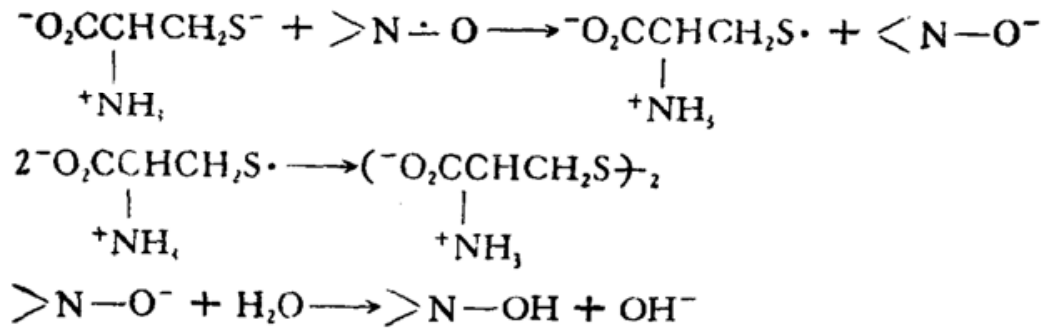

呱啶氮氧自由基与谷胱甘肽 $(\mathrm{GSH})$ 在不同的 $\mathrm{pH}$ 值的缓冲溶液中,在绝䂇、避光和室温 条件下,发生如下反应 ${ }^{[n}$ :

$$
\text { GSH }
$$

$\mathrm{pH}=2$ 时, 产物为相应的磺酸 $\left(\mathrm{GSO}_{3} \mathrm{H}\right) ; \mathrm{pH}-9.5$ 时, 产物为二硫化物 (GSSG); 当 pH介于 $2.5-8.5$ 时,生成两种产物的混合物. 
当 $\mathrm{pH}-2$ 时, 反应可能按下列方式进行:

$$
\begin{aligned}
& \text { 2 }>\mathrm{N}=\mathrm{O}+\mathrm{H}^{+} \longrightarrow \mathrm{N}^{+}=\mathrm{O}+>\mathrm{N}-\mathrm{OH} \\
& >\mathrm{N}^{+}=\mathrm{O}+\mathrm{GSH} \stackrel{\mathrm{H}_{2} \mathrm{O}}{\longrightarrow} \mathrm{GSO} \mathrm{H}_{3} \mathrm{H}+>\mathrm{N}-\mathrm{OH}
\end{aligned}
$$

在碱性条件下, 反应机理为 ${ }^{(6)}$ :

$$
\begin{aligned}
& \mathrm{GSH}+\mathrm{B}^{-} \rightleftarrows \mathrm{GS}^{-}+\mathrm{HB} \\
& \mathrm{GS}^{-}+>\mathrm{N}-\mathrm{O} \longrightarrow \mathrm{GS} \cdot+>\mathrm{N}-\mathrm{O}^{-} \\
& 2 \mathrm{GS} \longrightarrow \mathrm{GSSG} \\
& >\mathrm{N}-\mathrm{O}^{-}+\mathrm{HB} \rightleftharpoons \mathrm{N}-\mathrm{OH}+\mathrm{B}^{-}
\end{aligned}
$$

电子转移反应 (45)为速率决定步骤.

哌啶氮篻自由基和羟胺盐酸盐在酸性条件下迅速发生氧化还原反应:

$$
\text { 4> } \mathrm{N}-\mathrm{O}+2 \mathrm{NH}_{2} \mathrm{OH} \stackrel{\mathrm{PH}<5}{\longrightarrow} 4>\mathrm{N}-\mathrm{OH}+\mathrm{N}_{2} \mathrm{O}+\mathrm{H}_{2} \mathrm{O}
$$

哌啶氮氧自由基 II 与羟胺在不同的 $\mathrm{pH}$ 值条件下发生不同的反应:

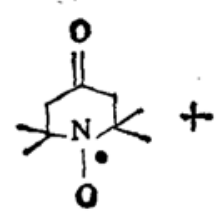

\section{$\mathrm{NH}_{2} \mathrm{OH} \cdot \mathrm{HCl}$}

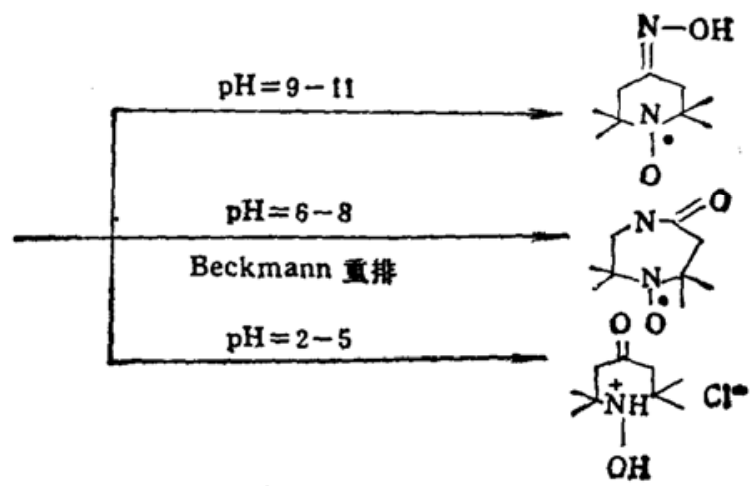

氮氧自由基与芳基重氮盐反应, 涉及一个初始的电子转移步骤:

$$
\mathrm{ArN}_{2}^{+}+\mathrm{N}>-\mathrm{O} \longrightarrow \mathrm{Ar} \cdot+\mathrm{N}_{2}+>\mathrm{N}^{+}=\mathrm{O}
$$

用激光照射二叔丁基氮氧自由基的四氯化碳溶液, 可发生如下的电子转移过程:

$$
\begin{gathered}
\left(\mathrm{Me}_{3} \mathrm{C}\right)_{2} \mathrm{~N}-\mathrm{O} / \mathrm{CCl}_{4} \stackrel{\mathrm{h} \nu}{\longrightarrow}\left[\left(\mathrm{Me}_{3} \mathrm{C}\right)_{2} \mathrm{~N}^{+}=\mathrm{O} / \mathrm{CCl}_{4}^{-}\right]^{*} \\
\longrightarrow\left(\mathrm{Me}_{3} \mathrm{C}\right)_{2} \mathrm{~N}^{+}=\mathrm{O} / \mathrm{Cl}^{-}+\mathrm{Cl}_{3} \mathrm{C}^{-} \\
\mathrm{Cl}_{3} \mathrm{C}^{*}+\left(\mathrm{Me}_{3} \mathrm{C}\right)_{2} \mathrm{~N}-\mathrm{O} \longrightarrow\left(\mathrm{Me}_{3} \mathrm{C}\right)_{2} \mathrm{NOCCl}_{3}
\end{gathered}
$$

Ingold ${ }^{\text {[n }}$ 证实了上述结果, 并发现哌啶氮氧自由基在二溴甲烷、碘甲烷等溶剂中存在电子 转移反应.

在 $\beta$-环糊精存在下, 氮氧自由基与维生素 $\mathrm{C}$ 的电子转移反应速率下降, 这是由于氮氧自 由基与 $\beta$-环糊精形成了包合物。研究表明,氮氧自由基在 $\beta$-环糊精存在下与维生素 $\mathrm{C}$ 的反 应按如下机理进行:

$$
\begin{aligned}
& >\mathrm{N}-\mathrm{O}+\mathrm{CD} \longrightarrow(>\mathrm{N}-\mathrm{O}-\mathrm{CD}) \\
& >\mathrm{N}-\mathrm{O}+\mathrm{HA} \longrightarrow>\mathrm{N}-\mathrm{OH}+\mathrm{A}^{:} \\
& (>\mathrm{N}-\mathrm{O}-\mathrm{CD})+\mathrm{HA}^{-} \longrightarrow>\mathrm{N}-\mathrm{OH}+\mathrm{A}^{-}+\mathrm{CD} \\
& \mathrm{A}^{-}+\mathrm{A}^{-} \longrightarrow \mathrm{A}+\mathrm{A}^{2-}
\end{aligned}
$$

氮氧自由基 I 和 III 与 $\mathrm{N}, \mathrm{N}, \mathrm{N}^{\prime}, \mathrm{N}^{\prime}$-四甲基对苯二胺的电子转移反应速率在环糊精存在 
下也有所降低 ${ }^{(8)}$.

Saint-Aman ${ }^{[9]}$ 用电化学方法研究了氮氧自由基与环糊精之间的相互作用.

哌啶氮氧自由基与维生素 $\mathrm{C}$ 在胶束体系中氧化还原反应研究结果表明, 氮氧自由基电子 转移反应速率受胶束性质和氮篻自由基亲脂性的影响, 正离子胶束（CTAB）可提高反应速 率,负离子胶束 (SDS) 降低反应速率,氮氧自由基的亲脂性越强, 这种作用就越明显. 电中 性的胶束对反应速率没有影响 ${ }^{[0]}$.

哌淀氮氧自由基的氧化产物氧铵盐具有很强的氧化性,能氧化醇、胺、酮、酚和膦等. 但与 烃类、芳醚、硫化物以及砐等不反应.

在弱碱性溶液中, 用电化学方法产生的哌啶氧铰盐可氧化醇、胺.

$$
\begin{aligned}
& >\mathrm{N}-\mathrm{O} \underset{+\mathrm{e}^{-}}{\stackrel{-\mathrm{e}^{-}}{\rightleftharpoons}}>\mathrm{N}^{+}=\mathrm{O} \\
& >\mathrm{N}^{+}=\mathrm{O}+\mathrm{RCH}_{2} \mathrm{OH} \longrightarrow \mathrm{RCHO}+>\mathrm{N}-\mathrm{OH}+\mathrm{H}^{+} \\
& >\mathrm{N}^{+}=\mathrm{O}+\mathrm{RCH}_{2} \mathrm{NH}_{2} \longrightarrow[\mathrm{RCH}=\mathrm{NH}]>+>\mathrm{N}-\mathrm{OH}+\mathrm{H}^{+} \\
& \quad>\mathrm{N}^{+}=\mathrm{O} \\
& \mathrm{RCH}=\mathrm{O} \longleftrightarrow \mathrm{RCN}+>\mathrm{N}-\mathrm{OH}+\mathrm{H}^{+} \\
& >\mathrm{N}-\mathrm{OH}+>\stackrel{+}{\mathrm{N}}=\mathrm{O} \longrightarrow 2>\mathrm{N}-\mathrm{O}+\mathrm{H}^{+}
\end{aligned}
$$

反应在较低的电势下发生, 不会将䤊进一步氧化为酸, 对一级醇具有很好的选择性.

在 $\mathrm{Cu}^{2+}$ 和 $\mathrm{O}_{2}$ 存在的条件下, 哌啶氮氧自由基能把一级醇篻化为醛,其中经历了氧铵盐 中间体。

$$
\begin{aligned}
& \mathrm{Cu}^{2+}+>\mathrm{N}-\mathrm{O} \longrightarrow \mathrm{Cu}^{+}+>\stackrel{+}{\mathrm{N}}=\mathrm{O} \\
& >\mathrm{N}^{+}=\mathrm{O}+\mathrm{RCH}_{2} \mathrm{OH} \longrightarrow>\mathrm{N}-\mathrm{OH}+\mathrm{RCH}=\mathrm{O}+\mathrm{H}^{+} \\
& >\mathrm{N}-\mathrm{OH}+>\mathrm{N}^{+}=\mathrm{O} \longrightarrow 2>\mathrm{N}-\mathrm{O}+\mathrm{H}^{+} \\
& 4 \mathrm{Cu}^{+}+4 \mathrm{H}^{+}+\mathrm{O}_{2} \longrightarrow 4 \mathrm{Cu}^{2+}+2 \mathrm{H}_{2} \mathrm{O}
\end{aligned}
$$

哌啶氧铵盐能将酮氧化成 $\alpha$-二酮:

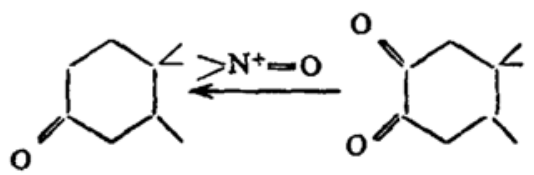

产物分析发现有哌啶氮羍自由基生成. 因此,反应可能涉及电子转移过程。羍铵盐可以氧 化胺 ${ }^{[1]}$, 其机理为<smiles>[R]N(C)c1ccccc1</smiles><smiles>[3H][N+](=O)[O-]</smiles><smiles>[R][N+]1(c2ccccc2)O[N+](C)(C)[CH][CH][C@H]1[2H]</smiles>

$$
>_{\mathrm{NH}}^{+} \rightarrow \mathrm{O}^{-}+{ }_{\mathrm{R}}^{\mathrm{Ph}} \underset{+}{\mathrm{N}}=\mathrm{CH}_{2}
$$




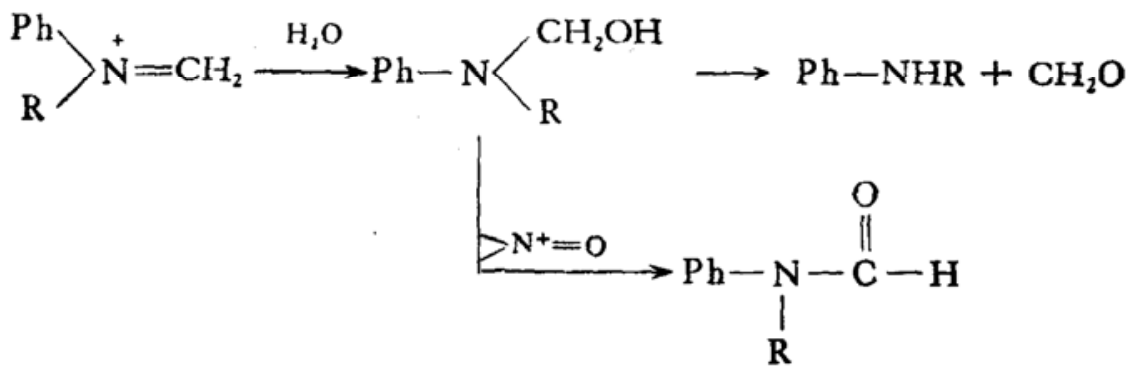

哌啶氧铵盐与半睄氨酸在室温、避光、绝氧条件下, 在 $\mathrm{HCl}$ 水溶液中反应, 生成哌啶差胺 和磺基丙氨酸 ${ }^{[12]}$.

$$
\begin{aligned}
& 3>\stackrel{+}{\mathrm{N}}=\mathrm{O}+\mathrm{HSCH}_{2} \mathrm{CH}\left(\mathrm{NH}_{2}\right) \mathrm{CO}_{2} \mathrm{H} \longrightarrow \\
& 3>\mathrm{N}-\mathrm{OH}+\mathrm{HO}_{3} \mathrm{SCH}_{2} \mathrm{CH}\left(\mathrm{NH}_{2}\right) \mathrm{CO}_{2} \mathrm{H}
\end{aligned}
$$

具有不同反离子的氧铵盐对醇的氧化反应具有不同的选择性.

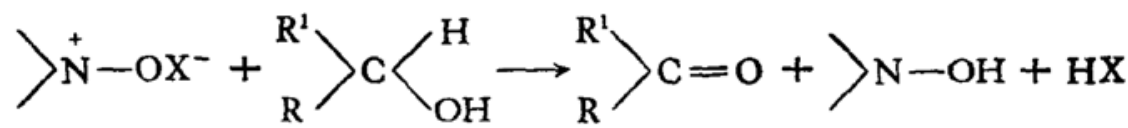

$\mathrm{X}-\mathrm{Br}(\mathrm{a}), \mathrm{Cl}(\mathrm{b}), \mathrm{R}^{1}-$ 烷基、 $\mathrm{H}$.

烯丙醇与 $\mathrm{a}$ 反应, 产物主要为卤代醇. 与 $\mathrm{b}$ 反应, 产物为 $\alpha, \beta$-不饱和酫. 用 $\mathrm{a}$ 氧化二级 醇的速度比氧化一级醇的速度块, 同 $\mathrm{b}$ 氧化一级醇的速度大于氧化二级醇的速度.

哌啶氮氧自由基在痕量强酸作用下能诱发过氧化物分解 ${ }^{[13]}$. 在 $\mathrm{HCl}$ 存在下, 氮氧自由 基歧化为羟胺和氧铵盐. 氧铵盐与烷基过氧化物作用,生成羟胺和烷基过氧自由基. 哌啶㻌. 铵盐可以氧化芐醇 ${ }^{[14]} 、$ 二元醇 ${ }^{[15]}$ 及其他有机物 ${ }^{[16]}$, 还可用来产生自由基正离子.

最近, 本研究室研究了用哌啶氧铵盐选择性地氧化一级醇、二级醇的反应 ${ }^{[17]}$, 发现反应的 选择性高、条件温和, 在合成上有实用价值. 2,2,6,6-四甲基-4-乙酰氧基哌啶氧铵六氯锑酸 盐可与 $\mathrm{N}$-烷基吩噻嗪衍生物反应, 得到相应的吩噻嗪衍生物自由基正离子盐, 方法简便, 产 物纯净, 并可以制成单晶 ${ }^{[18]}$. 哌啶氧铵盐与 $\beta$-环糊精可形成包合物, 这种包合物与 $\mathrm{N}$-取代 吩噻嗪 (取代基为一 $-\mathrm{CH}_{3},-\mathrm{CH}_{2} \mathrm{CH}_{3},-\mathrm{Ph},-\mathrm{CH}_{2} \mathrm{CH}\left(\mathrm{CH}_{3}\right)_{2}$ ) 的 $\beta$-环糊精包合物在水溶液 中发生电子转移反应,产生 $\beta$-环糊精包合的 $\mathrm{N}$-取代吩噻嗪自由基正离子 ${ }^{[19]}$. 哌啶氧铵盐可 以把胆红素氧化成相应的自由基正离子 ${ }^{[20]}$.

氮氧自由基是一类具有重要理论意义和应用价值的稳定自由基. 近年来, 氮氧自由基的 研究发展很快, 其中哌啶氮氧自由基的研究占有突出的地位. 由研究稳定氮氧自由基而发展 起来的自旋标记和自旋探针技术为研究化学体系和生物体系中的微观环境, 从分子水平上认 识生物有机体的复杂运动规律提供了有力的工具. 哌啶氮氧自由基与生物活性分子的单电子 转移反应研究对于从分子水平上认识生命过程中氧化还原反应具有一定意义. 哌啶氧铵盐作 为一类电子受体,可氧化醇、酚、胺和酮等化合物,还可用以产生臽由基正离子.

\section{参考文献}

[1] Liu, Y.C., Lhang, F., Jiang, Z. Q., Acta Chimica Sinica, 1987, 45: 477.

[2] Liu, Y. C., Zharg, F., Jiang, Z. Q., Acta Chimtca Sinica, 1989, 47: 1120.

[3] Fish, J. R., Swarts, S. G., Sevilla, M. D. et sl., J. Phys. Chem., 1988, 92: 3745.

[4] John, F. W., Vanice, F. L., Phys Chem. Phys. Med. NMR 1984, (16): 477; Belkin, S., Mehlhorg, R. J., Hideg, K. et al.. Arch. Biochem. Biofhys., 1987, 256: 232. 
[5]刘有成、江致勤、高忠理,科学通报, 1986,31(11): 878 .

[6] 刘有成、高忠理,科学通报, 1987,32(19): 1465 .

(7) Chateauneuf, J., Lusztyk. J., Ingold, K.U., J. Org. Chem., 199:), 55: 1061.

(8) Luwazi, 1., Michon, J., Rassat, A., New. J. Chem.. 1988, (12): 123.

19] Saint-Aman, E., Serve, D., New. J. Chem., 1989, (13): 121 .

[10] Liu. Y. C., Han, Z.X., Wu, L.M. et al.. Science in China, Ser. B, 1989, 32: 24.

[11] Hunter, D. H., Rocok, J.S., Rey, A. W. et al., J. Org. Chem., 1988, 53:1278.

[12] Liu, Y. C., Zhang, F., Acta Chimica Sinica, 1989, 47: 411.

[13] Dulog, L., Bleher, R., Macromol. Chem., 1986, 187: 2357.

[14] Miyazawa, T., Endo, T., J. Mol. Catal., 1988, 149: 31.

[15] Aneu, P. L., Banfi, S., Motanari, F. et al., J. Org. Chem., 1989, 92:2970.

\{16] Kartasheva, Z. S., Kasaikina, O.T., Gagarina, A.B., Kinet. Katal., 1989, 30: 326.

[17] Liu. Y. C., Liu, Z. L., Guo, H. X., Chem. J. Chin. Univ. (Engl. Ed.), 1988, 4: 90; Liu, Y. C., Guo, H. X., Liu, Z. L., Acta Chimica Sinica, 1991, 49: 187.

[18] Liu, Y. C., Ding, Y. B., Liu, Z. L., Acta Chimica Sinica, 1990, 48: 1199; Liu, Y. C., Ding, Y. B., Liu, Z. L., Jiegon Huaxue, 1989, 8: 140; Wang, Q. G., Liu, Y. C., Ding, Y. B. et al., Jiegow Hнахше, 1987, (7): 153.

(19) Guo, Q. X., Huan. P., Liu, B. et al., Chin. Chem. Lett., 1992, 3: 53.

(20] Guo, Q. X., Yang, L., Liu, B. et al., Chem. Res. Chin. Univ., 1992, 8: 301. 